\title{
Therapeutic Effects of Slotted Manuduction on Knee Extension Apparatus-induced Traumatic Adhesion
}

\author{
Qing Ye*, Chanjuan Ji, Jinliang Hu \\ Department of Operating, Binzhou People's Hospital, Binzhou, China
}

Email address:

yeqing2019@163.com (Qing Ye)

*Corresponding author

\section{To cite this article:}

Qing Ye, Chanjuan Ji, Jinliang Hu. Therapeutic Effects of Slotted Manuduction on Knee Extension Apparatus-induced Traumatic Adhesion. American Journal of Life Sciences. Vol. 7, No. 6, 2019, pp. 110-114. doi: 10.11648/j.ajls.20190706.12

Received: August 31, 2019; Accepted: October 26, 2019; Published: November 8, 2019

\begin{abstract}
Objective: To evaluate the therapeutic effects of slotted manuduction on knee extension apparatus-induced traumatic adhesion in multiple medical institutions. Methods: A total of 160 patients with knee extension apparatus induced traumatic adhesion who were admitted in our hospital from January 2017 to December 2017 were selected, and randomly divided into two groups: experimental group and control group, $(\mathrm{n}=80)$. There were 44 males and 36 females in the experimental group, with mean age of (45.95 \pm 9.48$)$ years old, which was treated with slotted manuduction; and 43 males and 37 females in the control group, with mean age of $(46.52 \pm 9.07)$ years old, which received traditional treatment. The treatment was performed once per day for 30 days, with 30 minutes once a time. Informed consent was obtained from each patient. Main outcome measures: The degree of knee joint activities, score of knee joint actively flexural behavior function, and the grading of joint function prior to and after treatment, as well as therapeutic effects were compared in two groups. Results: The significant effective rate was $90.00 \%$ in the experimental group and $76.25 \%$ in the control group $(\mathrm{P}<0.05)$, the former group more effective than the latter group. Comparing the degree of knee joint activities, score of knee joint function, score of knee joint actively flexural behavior function, and the grading score of joint function, the experimental group was better than the control group $(\mathrm{P}<0.01)$. Conclusion: Slotted manuduction has an extract and safe effect on treating knee extension apparatus induced traumatic adhesion, which has high operational and promotional value.
\end{abstract}

Keywords: Knee Extension Apparatus, Traumatic Adhesion, Slotted Manuduction, Clinical Evaluation

\section{Introduction}

Knee extension apparatus induced traumatic adhesion refers to fiber stiffness induced by trauma, manifested with varying degrees of genuflex functional limitation. It is a common sequela of upper knee periarticular fracture or trauma in clinic, which is caused by a long-time fixation for femoral condyle or tibial tubercle traction due to patella fracture, femoral shaft fracture, proximal tibia fracture, femoral condyle fracture, dislocation of knee joint and many other reasons, causing great difficulties to patients' life and work [1-4].

After several years of experience learned in clinical practice, we have explored a set of techniques of four-finger acupressure and extension genuflex for the treatment of knee extension apparatus induced traumatic adhesion. As this method is concluded on the basis of the inheritance of "slotted manuduction for the treatment of injury of knee seam tendon" of the renowned TCM doctor in China, Mr. Liu Shoushan, it is called "slotted manuduction".

\section{Materials and Methods}

\subsection{Subjects}

A total of 160 patients with knee extension apparatus induced traumatic adhesion who were admitted in our hospital from January 2017 to December 2017 were selected, and randomly divided into two groups using the random number method: experimental group and control group, $(n=80)$. There were 44 males and 36 females in the experimental group, with mean age of $(45.95 \pm 9.48)$ years old, which was treated with slotted manuduction; and 43 males and 37 females in the control group, with mean age of $(46.52 \pm 9.07)$ years old, 
which received traditional treatment. The completion of the experiment reached the required number of cases. The subjects signed informed consent.

\subsection{Diagnostic, Inclusion and Exclusion Criteria}

Diagnostic criteria: joint fracture and dislocation, postoperative articular adhesion, intra-articular malunion and long-term immobilization; it was clinically manifested with knee flexion-extension functional limitation to varying degrees, even rigidity; examination: hard, pliable and inelastic soft tissues around the knee, smaller patella activity and skin contracture; $\mathrm{X}$-ray examination showed osteoporosis, soft tissue calcification, bone deformities or no obvious abnormalities [5, 6].

Inclusion criteria: 1) patients whose ages were between 20 and 60 years old; 2 ) patients who met the diagnostic criteria; 3 ) within six months after joint immobilization; 4) patients who agreed to give up other drugs, physical therapy, acupuncture and other treatment measures in the course of treatment [7].

Exclusion criteria: 1) patients who were accompanied by severe cardiopulmonary disease, hypertension or psychiatric history; 2) patients who accepted other treatments which could affect the effects of indicators in this experiment; 3) patients with joint adhesion induced by inflammation, tuberculosis, rheumatoid arthritis and other diseases; 4) patients whose fracture around the knee did not heal; 5) patients who were complicated by gonarthromeningitis or joint swelling; 6) patients whose X-ray films showed other bone diseases [8].

\subsection{Treatment Methods}

Slotted manipulative steps and methods in the experimental group: 1) position: the patient lay in supine position, and the doctor stood on the side of affected knee; 2) massage the quadriceps femoris and peripatellar soft tissue with rolling, rubbing and kneading manipulations to fully relax the tissues around joint (10 $\mathrm{min})$, and then place thumbs and index fingers of both hands in the quadrangles of patella, push the patella left and right, up and down and rotationally 10 times respectively for $5 \mathrm{~min}$ in total; 3) four-finger acupressure method: press acupoints of Xuehai, Liangqiu, Dubi and Neixiyan respectively with thumbs and index fingers of both hands, apply force slowly until sour pain, heavy and flatulence could be felt in joint and its surrounding; once acupressure every 2 min for a total of 3 times; 4) extension genuflex: the patient was sitting, with knee joint beside the bed to allow the knee to hang down naturally, an assistant helped press on the knee and a doctor squatted down besides the affected limb, with one hand pressing the knee and the other hand on the ankle to gradually shake the knee joint under the role of limbs gravity so as to slowly release articular spasm and adhesion (5 min). The doctor sandwiched the shanks of the patient between his legs with his thigh, the assistant pulled and extended them relatively, the doctor held Neixiyan and Waixiyan by double thumbs, with the other four fingers beneath the legs, joined palms holding the knee to gradually make its flexion as much as possible, which was repeated 8 to 10 times (10 min). The key points of this manuduction were that extension was continued and powerful to make knee seam open, and genuflex was gentle and strong within the patient's tolerance degree; 5) end manuduction: relax for $5 \mathrm{~min}$ by patting, striking, smoothing and straightening quadriceps femoris and other techniques [9].

Manipulative steps and methods in the control group: 1) posture: the patient lay in supine position, and the doctor stood on the side of affected knee; 2) massage from quadriceps femoris to tibial tubercle with both hands, massage quadriceps femoris group by relaxing practices, and push patella left and right, up and down 10 times respectively; then, do the moving-aside test, drawer test and Mcmurray's test on the affected knee 3 times respectively; 3) raise the straight leg of patient by $45^{\circ}$, support the distal thigh with one hand to make shanks hang down naturally, slightly put pressure with the other hand; then complete flexion of the hip joint was made, the doctor put one hand on the knee, and the other hand held the ankle to put pressure for flexion; strengthen flexion training when extensor muscles were in a relaxing state. This manuduction was repeated 8 to 10 times; 4) the patient was in a prostrate position, support pads put in the lower abdomen and before hip; the doctor pressed the distal thigh with one hand, the other hand held the ankle to put pressure for flexion to gradually transit to a genuflex sitting state to close to the knee-chest prone position This manuduction was repeated 8 to 10 times; 5) end manuduction: relax for $5 \mathrm{~min}$ by patting, striking, smoothing and straightening quadriceps femoris and peripatellar soft tissue [10].

Treatment in the two groups was once per day, $30 \mathrm{~min}$ for each time, with 10 times as a course of treatment for a total of three courses. The interval between two courses treatment was $3 \mathrm{~d}$.

Clinical criteria: 1) clinical recovery: range of joint motion $\geq$ $100^{\circ}$, score of knee joint function was increased by 10 points or above. 2) significant effectiveness: range of joint motion between $80^{\circ}$ and $100^{\circ}$, and score of knee joint function was increased by 10 points or above; 3 ) effectiveness: range of joint motion between $60^{\circ}$ and $80^{\circ}$, or $<60^{\circ}$, score of knee joint function was increased by 6 points or above; 4 ) ineffectiveness: joint activity had no significant improvement, and score of knee joint function was increased by less than 6 points [11].

Main outcome measures: The degree of knee joint activities, score of knee joint actively flexural behavior function and grading of joint function prior to and after treatment, as well as therapeutic effects were compared in two groups.

\subsection{Statistical Analysis}

All data were analyzed by SPSS 14.0. Balance test: $\chi^{2}$ test, $\mathrm{t}$ test and rank-sum test; clinical therapeutic effect analysis: measurement data were subjected to paired-sample rank-sum test; comparison across groups: categorical data were subjected to $\mathrm{CMH}$ Chi-square test; measurement data were subjected to rank-sum test. Unilateral test was used, with $\mathrm{P}<0.05$ being statistically significant.

\section{Results}

\subsection{Baseline Data Comparison Between Two Groups}

The comparison of the parts of fracture between the two 
groups was shown in Table 1.

Table 1. Fracture part comparison (n/\%).

\begin{tabular}{|c|c|c|c|c|c|c|c|}
\hline Group & Patella & Femoral condyle & Femur trunk & Femoral neck & Femur section & $\chi^{2}$ & $\mathbf{P}$ \\
\hline Experimental & $26 / 32.50$ & $17 / 21.25$ & $16 / 20.00$ & $13 / 16.25$ & $8 / 10.00$ & 0.627 & 0.903 \\
\hline Control & $25 / 31.25$ & $19 / 23.75$ & $15 / 18.75$ & $14 / 17.5$ & $7 / 8.75$ & 0.627 & 0.903 \\
\hline
\end{tabular}

The comparison of the degree of knee joint activities, grading of joint function and score of knee joint actively flexural behavior function prior to treatment between the two groups was shown in Table 2.

Table 2. Comparison between degree of knee joint activities, grading of joint function and score of knee joint actively flexural behavior function prior to treatment.

\begin{tabular}{lllll}
\hline Group & $\mathbf{n}$ & Degree of knee joint activities $\left.\mathbf{(}^{(}\right)$ & Score of knee joint actively flexural behavior function & Grading of joint function \\
\hline Experimental & 80 & $6.28 \pm 2.16$ & $5.71 \pm 0.84$ & $3.68 \pm 0.54$ \\
Control & 80 & $10.32 \pm 2.41$ & $5.43 \pm 0.88$ & $3.52 \pm 0.46$ \\
z/t & & 2.49 & 2.81 & 0.42 \\
$\mathrm{P}$ & 0.0861 & 0.0206 & 0.5291 \\
\hline
\end{tabular}

\subsection{Main Outcomes Measures After Treatment}

The comparison of the degree of knee joint activities between the two groups was shown in Table 3.

Table 3. Degree of knee joint activities after treatment.

\begin{tabular}{lllllll}
\hline Group & \multirow{n}{*}{ Before } & \multirow{2}{*}{ After } & Difference & \multicolumn{2}{l}{ Self-comparison before and after treatment } \\
Experimental & 80 & $4.28 \pm 0.61$ & $2.01 \pm 0.59$ & $-2.27 \pm 0.60$ & -1843.6 & 0.000 \\
Control & 80 & $4.21 \pm 0.68$ & $2.84 \pm 0.56$ & $-1.37 \pm 0.62$ & -1568.5 & 0.002 \\
Z & & 87.34 & & & & \\
P & & 0.001 & & & & \\
\hline
\end{tabular}

The comparison of score of knee joint actively flexural behavior function between the two groups was shown in Table 4 .

Table 4. Score of knee joint actively flexural behavior function after treatment.

\begin{tabular}{lllllll}
\hline \multirow{2}{*}{ Group } & \multirow{2}{*}{ Before } & \multirow{2}{*}{ After } & Difference & \multicolumn{2}{c}{ Self-comparison before and after treatment } \\
\cline { 5 - 7 } & & & & Z & P \\
Experimental & 80 & $5.16 \pm 0.64$ & $2.92 \pm 0.69$ & $-2.24 \pm 0.65$ & -1795.4 & 0.000 \\
Control & 80 & $4.95 \pm 0.60$ & $3.24 \pm 0.57$ & $-1.71 \pm 0.58$ & -1658.7 & 0.001 \\
Z & & 78.66 & & & & \\
P & & 0.000 & & & & \\
\hline
\end{tabular}

The comparison of grading of joint function between the two groups was shown in Table 5.

Table 5. Grading of joint function after treatment.

\begin{tabular}{lllllll}
\hline Group & $\mathbf{n}$ & Before & \multirow{2}{*}{ After } & Difference & \multicolumn{2}{c}{ Self-comparison before and after treatment } \\
\cline { 5 - 7 } & & & & $\mathbf{Z}$ & \multicolumn{1}{c}{} \\
\hline Experimental & 80 & $3.32 \pm 0.41$ & $2.16 \pm 0.38$ & $-1.16 \pm 0.40$ & -1135.5 & 0.002 \\
Control & 80 & $3.28 \pm 0.42$ & $2.47 \pm 0.43$ & $-0.81 \pm 0.42$ & -1016.8 & 0.003 \\
Z & & 33.86 & & & & \\
P & & 0.003 & & & & \\
\hline
\end{tabular}

The comparison of TCM therapeutic effects between the two groups was shown in Table 6.

Table 6. Therapeutic effects of two groups.

\begin{tabular}{lllllll}
\hline Group & $\mathbf{n}$ & Effective (n/\%) & Ineffective (n/\%) & Effective rate (\%) & CMH & P \\
\hline Experimental & 80 & $72 / 90.00$ & $8 / 10.00$ & 90.00 & \multirow{2}{*}{0.821} & 0.004 \\
Control & 80 & $61 / 76.25$ & $19 / 23.75$ & 76.25 & \\
\hline
\end{tabular}

CMH (Cochran-Mantel-Haenszel)

The results showed that the baseline of both groups was consistent before treatment, which was comparable. Compared with traditional treatment in the control group, slotted manuduction for the treatment of knee extension apparatus induced traumatic adhesion could more effectively increase the degree of knee joint activities, improve knee joint function and significantly enhance the effective rate of treatment, between which the difference was statistically 
significant $(\mathrm{P}<0.01)$.

\section{Discussion}

The non-surgical treatments for this disease or postoperative rehabilitation therapies in clinic include massage, Chinese medicine fumigation, continuous traction, peroral Chinese herbs, muscle functional training, continuous passive motion (CPM) and passive training, etc., most of which, as one of combined therapies of traditional Chinese medicine, can be found in various types of research. Manipulative therapy is considered to be a method playing a key role in the Chinese medicine treatment. The Chinese medicine treatment for this disease is mainly based on the etiology and pathogenesis combining syndrome differentiation, and this disease is often treated by relaxing tendon and activating collaterals, promoting blood circulation to remove blood stasis, resolving hard lump and easing joint movement. Manipulation refers to do various actions in specific parts or acupuncture points on a patient's body surface with the hands of doctor to regulate the body's physiological and pathological states by the strength and skill of hands so as to reach therapeutic purposes. The effects of manipulative therapy for tendon injuries are mainly to relax tendon and activate collaterals, adjust suture, relieve spasm, relax muscles, release adhesions, lubricate joint, and regulate qi and blood $[12,13]$.

Modern medical research shows that the major pathological changes of knee extension apparatus induced adhesion: 1) quadriceps femoris and its tendon contracture occurs. 2) Patellar retinaculum has contracture and adhesion with femoral condyle. 3) Vastus intermedius fibrosis makes the activities between quadriceps femoris and its tendon and femur disappear. 4) Rectus femoris is shortened [14, 15]. Only by removing all factors that hinder flexion activities can the knee restore satisfied function. Therefore, foreign scholars proposed quadriceps femoris plasty for the treatment of knee extension apparatus induced adhesion, and on this basis, domestic scholars put forward the surgical method of small-incision knee joint adhesion lysis [16]. But whether it is classic or improved open lysis or arthroscopic lysis, it is generally considered to have many postoperative complications, such as knee extension apparatus injury, skin damage, local hematoma formation and infection, neurovascular injury, weakened knee extension function and walking fatigue, etc. [17].

Traditional Chinese medicine holds that knee extension apparatus induced adhesion is mainly caused by long-term inappropriate external fixation for bone fracture or other diseases related to the knee to result in osteocomma akinesia, qi and blood stasis, meridian blocking and unsmooth body fluid transportation, so that muscles, bones and joints lose the nourishment of qi-blood and fluid and exogenous pathogenic factors of wind, cold and dampness take the disadvantage of invasion, which leads to muscle and tendon atrophy and joint stagnation over time [18, 19].

The release practices in the beginning and relaxation practices at the end of slotted manuduction are basically the same as conventional techniques, the difference between which lies in four-finger acupressure and extension genuflex that play a key role in improving joint function. The four-finger acupressure in slotted manuduction means to press four acupoints in the meridian system, i.e. Liangqiu, Dubi, Xuehai and Neixiyan at the same time with thumbs and index fingers of both hands, which can dredge meridian qi and blood, coordinate the internal and external balance of muscles and joints, promote local qi-blood circulation, enhance metabolism, and release local injured tissues. In this study, the slotted manuduction therapy both stresses the importance of the practices of and quotes the meridian theory in traditional Chinese medicine, obtaining a great effect by a simple method [20]. It can be seen from the results of the two groups that slotted manuduction for the treatment of knee extension apparatus induced traumatic adhesion can better improve degree of knee joint activities than traditional means, which is in line with clinical practices, and has obtained a better therapeutic effect.

\section{Conclusion}

Extension genuflex can pull knee seam open by extended traction, thus contributing to the restoration of normal knee function, and then passive knee flexion can effectively release spasm and adhesion of knee joint and periarticular tissues. Since this operation is conducted after acupressure, periarticular tissue spasm has been partially lifted, so patients tend not to feel the pain, which is easier to remove tissue spasm and adhesion without pain and restore joint function.

\section{References}

[1] Rosenberg AG. Management of extensor mechanism rupture after TKA. J Bone Joint Surg Br. 2017; 94 (11): 116-9.

[2] Whiteside LA. Surgical technique: vastus medialis and vastus lateralis as flap transfer for knee extensor mechanism deficiency. Clin Orthop Relat Res. 2017; 471 (1): 221-30.

[3] Verbist DE, Boyd TG, Malkani AL, Wilhelmi BJ. CASE REPORT Simultaneous Latissimus Dorsi Myocutaneous Flap Transfer and Revision Total Knee Arthroplasty With Allograft Extensor Mechanism Reconstruction: A Case Report. Eplasty. 2017; 12 (1): 43-6.

[4] Holzapfel BM, Pilge H, Toepfer A, Jakubietz RG, Gollwitzer H, Rechl H, von Eisenhart-Rothe R, Rudert M. [Proximal tibial replacement and alloplastic reconstruction of the extensor mechanism after bone tumor resection]. Oper Orthop Traumatol. 2017; 24 (3): 247-62.

[5] Mavrogenis AF, Angelini A, Pala E, Sakellariou VI, Ruggieri P, Papagelopoulos PJ. Reconstruction of the extensor mechanism after major knee resection. Orthopedics. 2017; 35 (5): 672-80.

[6] Gregory JM, Sherman SL. Patellar stress fracture after transosseous extensor mechanism repair: report of 3 cases. Am J Sports Med. 2017; 40 (7): 1668-72. 
[7] Erpelding JM, Mailander A, High R, Mormino MA, Fehringer EV. Outcomes following distal humeral fracture fixation with an extensor mechanism-on approach. J Bone Joint Surg Am. 2017; 94 (6):548-53. Berend ME. Evaluation and management of complications of the extensor mechanism. Instr Course Lect. 2017; 61: 405-9.

[8] Burnett RS, Berger RA, Della Valle CJ, Sporer SM, Jacobs JJ, Paprosky WG, Rosenberg AG. Extensor mechanism allograft reconstruction after total knee arthroplasty. J Bone Joint Surg Am. 2005; 87 Suppl 1(Pt 2): 175-94.

[9] Duchman K, Mellecker C, Thedens DR, Albright JP. Quantifying the effects of extensor mechanism medialization procedures using MRI: a cadaver-based study. Iowa Orthop J. 2017; 31: 90-8.

[10] Kumar AR, Hunt P. Successful knee extensor mechanism reconstruction in a warfare-related open lower extremity injury complicated by mucormycosis infection: a case report. J Orthop Trauma. 2017; 26 (2): 7-10.

[11] Namdari S, Milby AH. Limb salvage after infected knee arthroplasty with bone loss and extensor mechanism deficiency using a modular segmental replacement system. J Arthroplasty. 2019; 26 (6): 977-81.

[12] Ek EW, Rozen WM. Surgical options for reconstruction of the extensor mechanism of the knee after limb-sparing sarcoma surgery: an evidence-based review. Arch Orthop Trauma Surg. 2019; 131 (4): 487-95.

[13] Bali K, Mootha AK, Krishnan V, Kumar V, Rawall S, Goni V.
A rare case of bicondylar Hoffa fracture associated with ipsilateral tibial spine avulsion and extensor mechanism disruption. Chin J Traumatol. 2017; 14 (4): 253-6.

[14] Lewullis GE, Jasko JG. Revision extensor mechanism allografting after total knee arthroplasty. Am J Orthop (Belle Mead NJ). 2017; 39 (11): 539-42.

[15] Durand S, Gaujoux G, Macquillan A. Triggering of the lateral slip of the extensor mechanism on a Bouchard's node. J Hand Surg Eur Vol. 2019; 36 (4): 340-1.

[16] Tuong B, White J. Get a kick out of this: the spectrum of knee extensor mechanism injuries. Br J Sports Med. 2019; 45 (2): $140-6$.

[17] Wright RC, Gillis CT, Yacoubian SV, Raven RB 3rd, Falkinstein Y, Yacoubian SV. Extensor mechanism repair failure with use of bidirectional barbed suture in total knee arthroplasty. J Arthroplasty. 2017; 27 (7): 1-4.

[18] Drexler M, Blumberg N. Extensor mechanism reconstruction: a novel technique for bone patella allograft post partial patellectomy failure. J Knee Surg. 2017; 24 (2): 129-35.

[19] Yoshida Y, Osaka S, Ryu J. Reconstruction of the knee extensor mechanism in patients with a malignant bone tumor of the proximal tibia. Surg Today. 2018; 40 (7): 646-9.

[20] Zwolak P, Kuhnel SP. Extraarticular knee resection for sarcomas with preservation of the extensor mechanism: surgical technique and review of cases. Clin Orthop Relat Res. 2019; 469 (1): 251-6. 